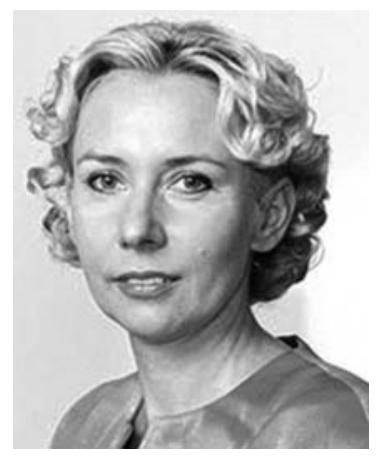

\title{
A Conversation with Joanna Wysocka
}

\author{
INTERVIEWER: EyTAN ZLOTORYNSKI
}

\author{
Senior Editor, Nature Reviews: Molecular Cell Biology
}

Joanna Wysocka is a Professor in the Departments of Chemical and Systems Biology and of Developmental Biology at Stanford University.

Eytan Zlotorynski: What are the general characteristics of the epigenetic modulation of enhancers during early embryogenesis?

Dr. Wysocka: Active enhancers have distinct epigenomic features that have been studied over the years, first in the context of individual loci and, more recently, across the genomes. Because to bind enhancer DNA, transcription factors have to compete out nucleosomes, the chief chromatin characteristic of enhancers is loss of nucleosomal density. Furthermore, active enhancers are associated with presence of general coactivator complexes and enrichment of certain histone modifications on the flanking nucleosomes. These simple combinatorial chromatin signatures interrogated via epigenomic approaches allow for genome-wide and cell type-specific enhancer mapping.

Eytan Zlotorynski: Can you explain to us the role of neural crest cells in embryogenesis and why you're studying them?

Dr. Wysocka: These cells are truly fascinating. They form early in development in the dorsal part of the neural tube. Then they delaminate and migrate long distances. They're sometimes referred to as "explorers of the embryo." A feature that initially attracted me to these cells is their enormous developmental plasticity. They can differentiate into over a hundred different cell types, including neurons and glia and a variety of mesenchymal cell types and even pigment cells. What is really interesting is that the cells are ectodermal in origin. They come from the neural tube, yet they're able to make all these mesenchymal derivatives that comprise our head and face. In fact, most of the head and face structures - bone, cartilage, connective tissue - are derived from the neural crest, whereas everywhere else in the body bone and cartilage are derived from the mesoderm. There have to be some interesting regulatory principles governing this unusual cellular plasticity.

Eytan Zlotorynski: You studied this with an evolutionary view by comparing human neural crest cells to chim- panzee neural crest cells to see the differences between them, especially with regard to how epigenetic modulations of enhancers contributed to craniofacial development. What were your major findings?

Dr. Wysocka: Neural crest cells are interesting from an evolutionary perspective because they are relatively late evolutionary inventions specific to vertebrates. They are thought to have facilitated our evolutionary success as vertebrates, because invention of the neural crest-derived structures such as jaws allowed for colonizing distinct environments and adapting to different predatory lifestyles. With respect to human evolution, neural crestderived structures are a strong target of selective pressure and contributed to human traits in multiple ways. They're responsible not only for the uniquely human facial appearance, but also for adaptations associated with enlarged brain size, speech articulation, upright posture. However, because of their transient nature - they form at 3-6 weeks of gestation - embryonic neural crest cells from humans and higher primates are largely inaccessible for studies. Our only way to really get a hold of these cells was to derive them in vitro from pluripotent stem cells. Once we developed and characterized this system for humans, we realized we could extend this approach to nonhuman primates and use comparative epigenomics to develop an evo-devo model of enhancer landscape evolution.

We used epigenetic profiling strategies to map enhancers both in human and chimpanzee neural crest to identify elements that are human-biased or chimp-biased in activity. This allowed us to systematically identify enhancer regions that change the regulatory activity during recent human evolution. Many of the enhancers that we find are associated with loci that have been implicated in craniofacial development, often with dosage-dependent effects as suggested by the haploinsufficiency in human syndromes. We're now exploring some specific candidates and their impact on neural crest cell behavior and morphology.

Eytan Zlotorynski: This variability in the activity of enhancers and their genes between chimpanzees and hu- 
mans is one of the bases for the phenotypic variability between us and other primates, and also between different human individuals, I presume. In this regard, which has the greater variability? Is it between chimpanzees and humans or between different human individuals? Or between different chimpanzees?

Dr. Wysocka: We're very interested in pursuing the intraspecies and individual variations of enhancer functions in the neural crest. Clearly, the differences between human and chimp are bigger. Also, the genomic variation is about 10 times bigger between chimp and human than even between human and Neanderthal. We have some preliminary data suggesting that overlapping sets of loci could be involved in both inter- and intraspecies variations of neural crest regulatory landscapes in higher primates.

Eytan Zlotorynski: Are you interested in exploring other morphogenetic developmental programs or is the primary goal to elucidate what makes us human or at least what makes us look human?

Dr. Wysocka: We're really focusing on the human face and the neural crest derivatives, because the complexity is so great it will take us years to decipher it. However, I think our approach, which I refer to as "cellular anthropology," meaning using in vitro differentiation models from higher primates to obtain and study evolutionarily important cell populations, will be broadly generalizable and can facilitate studies of regulatory evolution in many different systems and organs, including neural development.
Eytan Zlotorynski: Following the genome-wide demethylation in early human embryogenesis, several specific human endogenous retroviruses produce proteins and these proteins have cellular functions. Could you speculate what role such proteins, and human retroviruses in general, can have on human evolution and might still have on human phenotypic viability?

Dr. Wysocka: Probably the most prevalent way by which endogenous retroviruses contribute to human evolution is by contributing regulatory sequences like the LTR (long terminal repeat) elements. It's clear from multiple studies of different endogenous retroviruses that different LTRs can be exapted for regulatory functions, either as alternative promoters or as long-range enhancers. Although we don't see retroviral reactivation per se in neural crest cells and we don't see derepression in proteins being produced, we do see a subclass of the LTR elements from endogenous retroviruses being adapted for regulatory functions. Interestingly, some of our human-bias or chimp-bias enhancers are actually derived from the LTRs of endogenous retroviruses. Half of the human genome is composed of transposon elements and most of those transposons invaded the human genome at some point during primate evolution. They're not shared with the mouse, even though mice have their own set of transposons. If we want to understand the human- or primatespecific aspects of development, we simply cannot ignore half of the genome that is a playground for evolving new regulatory function. 


\section{$\$_{\text {CSH }}^{\infty}$ Cold Spring Harbor Symposia SYMPOSIA On Quantitative Biology}

\section{A Conversation with Joanna Wysocka}

Cold Spring Harb Symp Quant Biol 2015 80: 351-352

Access the most recent version at doi:10.1101/sqb.2015.80.030148

\section{License}

Email Alerting Receive free email alerts when new articles cite this article - sign up in Service the box at the top right corner of the article or click here. 